without needing a new experimental characterization.

\title{
References
}

[1] J. E.H. Tattersall , I. R. Scott , S. J. Wood , J. J. Nettell , M. K. Bevir , Z. Wang, N. P. Somasiri, X. Chen, Brain Research, vol. 904, pp. 43-53, 2001.

[2] A. G. Pakhomov, J. Doyle, B.E. Stuck, and M. R. Murphy, Bioelectromagnetics, vol. 24, pp. 174-181, 2003.

[3] A. Paffi, M. Pellegrino, R, Beccherelli, F. Apollonio; M. Liberti, D. Platano, G. Aicardi, G. D'Inzeo, submitted to IEEE Trans. Microwave Theory Tech..

[4] M. Pellegrino, A. Paffi, R. Beccherelli, M. Liberti, F. Apollonio, G. d'Inzeo, proceedings of Bioelectromagnetics 2005, Dublin, June 20-24.

Acknowledgements. This work was supported by the European Union, V framework under the RAMP2001 Project.

\section{* P-6 Statistical MUltipath Exposure OF A HUMAN IN A REALISTIC ELECTROMAGNETIC ENVIRONMENT}

Gunter Vermeeren, Wout Joseph, Christof Olivier, Luc Martens

Ghent University, Ghent, Belgium

Objectives. Several statistical models exist for the description of a realistic electromagnetic environment. If we want to investigate the whole-body exposure of a spheroid humanbody model in such an environment, then a huge number of simulations are required with any FDTD- or MoM/FEM tool in order to obtain statistical relevant results. Therefore, a new and fast method is proposed for the assessment of the exposure of a spheroid phantom in a realistic electromagnetic environment.

Methods. In [1] a statistical model of the electromagnetic field in an observation point $\mathbf{r}$ has been proposed for several realistic environments. This statistical model describes the electromagnetic field distribution as a finite sum of incident plane waves. Using this model, we generate a large number (50000) of complex field distributions.

To investigate the whole-body averaged SAR $\left(\mathrm{SAR}_{w b}\right)$ in any phantom, it is sufficient to determine the Poynting vector from the electric and the magnetic field distribution on a closed surface around the phantom. Integrating the Poynting vector over the observation surface and taking the real part, gives us the absorbed power inside the observation surface. As only the phantom is inside the observation surface, the absorbed power equals the losses in the spheroid phantom. Taking into account the weight of the spheroid phantom, one finds $\mathrm{SAR}_{w b}$.

The linearity of the Maxwell equations allows us to evaluate the fields on the observation surface for each individual incident plane wave and, finally, to make the superposition of the fields on this surface to find the resulting field. 
Because a homogeneous spheroid model has rotation symmetry around the major axis and reflection symmetry across the plane defined by the minor axes, the field distribution of any incident plane wave can be determined from the field distributions of the TE (transverse electric) and TM (transverse magnetic) polarized incident plane waves for every elevation angle $\theta$ between 0 and $\pi / 2$, and a single azimuth angle $\phi$ (see Fig. 1). The fields of these incident TE and TM polarized plane waves form a complete set of basis field distributions and have to be calculated with a FDTD or MoM/FEM tool.

The amount of elevation angles for which the fields of the TE and TM incident plane waves have to be calculated can be significantly reduced by the use of spline interpolation. With this method we have limited the number of simulations to 18 for the investigation of $\mathrm{SAR}_{w b}$ in a homogeneous spheroid in a realistic electromagnetic environment.

Using this set of 18 simulations of the fields and the interpolation, the absorption can be calculated from every complex field distribution generated with the model of [1].

Results. The proposed method was validated with a MoM/FEM tool. All deviations on $\mathrm{SAR}_{w b}$ were all below $1 \%$. After performing the numerical simulations to determine the set of basis field distributions, calculating the exposure for a single sample only takes 0.5 sec on a $\mathrm{PC}$ with a $3.4 \mathrm{GHz}$ processor. Fig. 2 shows the cumulative distribution function of $\mathrm{SAR}_{w b}$ in a homogeneous spheroid $(2 \mathrm{a}=1.75 \mathrm{~m}, \mathrm{~b}=0.138 \mathrm{~m})$ with dielectric properties $\epsilon_{r}=41.4$ and $\sigma=0.99 \mathrm{~S} / \mathrm{m}$ in an urban macrocell environment [1] at a frequency of $950 \mathrm{MHz}$. The number of samples was 50000. $\mathrm{E}_{r m s}$ averaged over free space was set to the ICNIRP reference level at $950 \mathrm{MHz}$, i.e. $42.38 \mathrm{~V} / \mathrm{m}$. For an urban macrocell the median of the $\mathrm{SAR}_{w b}$ is $0.014 \mathrm{~W} / \mathrm{kg}$ with standard deviation of $0.0025 \mathrm{~W} / \mathrm{kg}$. In $99 \%$ of the cases the $\mathrm{SAR}_{w b}$ will be less than $0.02 \mathrm{~W} / \mathrm{kg}$. The chance of reaching the ICNIRP limit of $0.08 \mathrm{~W} / \mathrm{kg}$ is not probable.

Conclusions. A new and fast method has been proposed for the investigation of the wholebody exposure of a spheroid model of a human in a realistic environment. The method has been validated with numerical simulations with a MoM/FEM tool and excellent agreement has been observed. The results of $\mathrm{SAR}_{w b}$ for a spheroid in an urban macrocell environment at a frequency of $950 \mathrm{MHz}$ are presented and are compliant with the basic restriction of $0.08 \mathrm{~W} / \mathrm{kg}$.

References:

[1] G. Vermeeren, C. Olivier, W. Joseph, and L. Martens, "Statistical model of the electromagnetic fields in a realistic environment", BEMS annual meeting, Japan, 2007. 


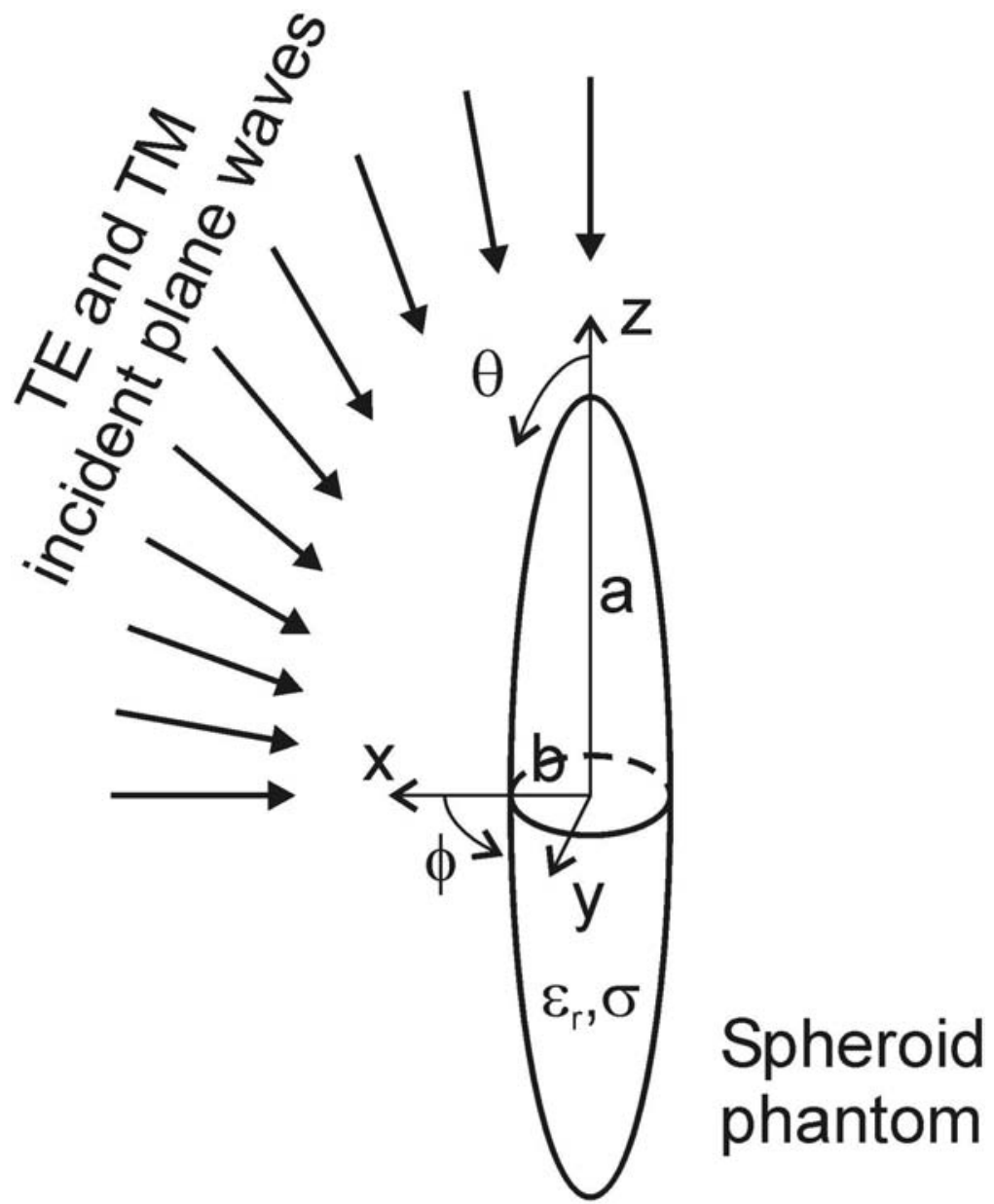

FiguRE 1.: The method for the exposure of a spheroid in a complex electromagnetic environment. 


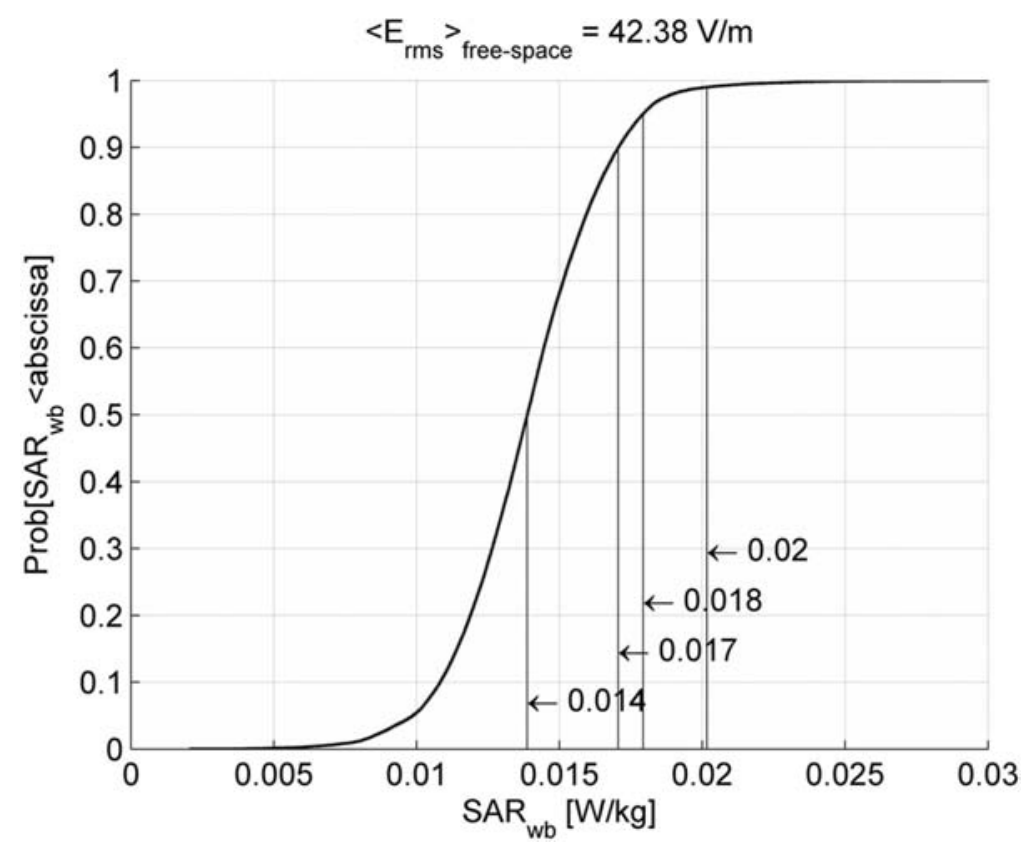

Figure 2.: The cumulative distribution function of $\mathrm{SAR}_{w b}$ in a spheroid model of a human $(2 \mathrm{a}=1.75 \mathrm{~m}, \mathrm{~b}=0.138 \mathrm{~m})$ in an urban macrocell environment at $950 \mathrm{MHz}$ when the average of $\mathrm{E}_{r m s}$ over the entire space equals the ICNIRP reference level. 


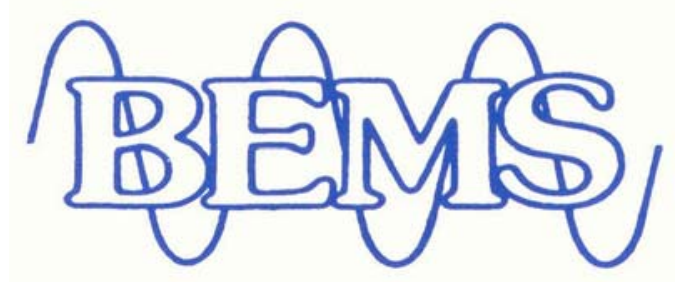

\section{The Bioelectromagnetics Society $29^{\text {th }}$ Annual Meeting Abstract Collection}

\section{Kanazawa-shi Bunka Hall Kanazawa, Japan June 10 - 15, 2007}

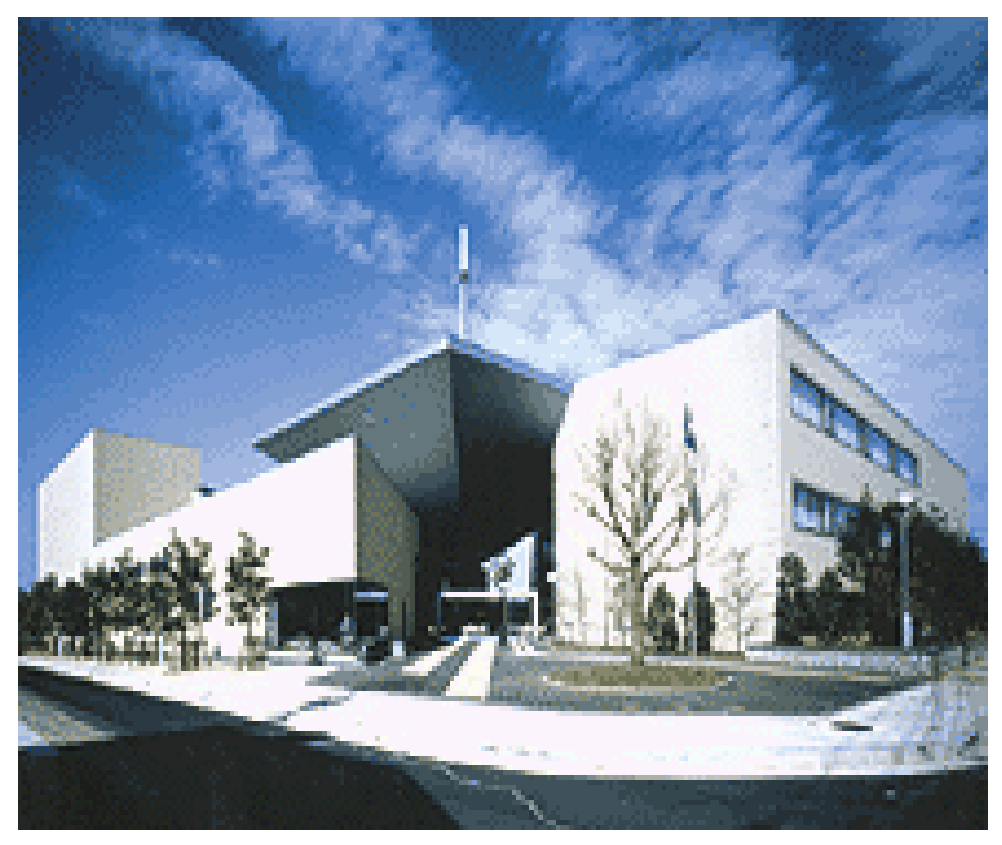

